\title{
Nonrelativistic variational calculations of the positronium molecule and the positronium hydride
}

\author{
Sergiy Bubin ${ }^{1}$ and Ludwik Adamowicz ${ }^{1,2}$ \\ ${ }^{1}$ Department of Chemistry, University of Arizona, Tucson, Arizona 85721, USA \\ ${ }^{2}$ Department of Physics, University of Arizona, Tucson, Arizona 85721, USA
}

(Received 3 October 2006; published 15 November 2006)

\begin{abstract}
Highly accurate ground-state nonrelativistic variational calculations of $\mathrm{Ps}_{2},{ }^{1} \mathrm{HPs}$, and ${ }^{\infty} \mathrm{HPs}$ are reported. The calculations have been performed using 5000 explicitly correlated Gaussian basis functions and yielded the lowest variational energy upper bounds for these systems to date. The relative accuracies of the energies obtained are of the order of $4 \times 10^{-10}$ a.u. for $\mathrm{Ps}_{2}$ and $2 \times 10^{-9}$ a.u. for HP. Several expectation values have also been computed for each system, as well as electron-positron annihilation rates.
\end{abstract}

DOI: 10.1103/PhysRevA.74.052502

PACS number(s): 36.10.Dr, 31.15.Ar

Since the theoretical prediction of their stability $[1,2]$, the positronium molecule and the positronium hydride have been subject to numerous theoretical studies (see, for example, Refs. [3-16], and references therein). The interest in these systems has increased in the last decade due to rapidly growing computer capabilities that have allowed treatment of four-particle and even larger quantum systems with high accuracy and due to the experimental observations of several positronic systems including HPs [17]. The positronium hydride and, especially, the positronium molecule are important systems among four-particle systems with Coulombic interactions. They are of great interest in astrophysical applications and in solid-state physics. Thus, very accurate solutions of the Schrödinger equation for the stationary bound states of these systems and predictions of expectation values related to measurable quantities are desirable. It should be noted that obtaining precise binding energies with many converged significant figures in the calculations is not only of purely technical interest. Very well converged calculations are often needed to obtain adequate precision in determining expectation values of some operators related to quantities other than the total energy of the system. Those expectation values may converge much slower than the total energy. This happens because the weight assigned by the operators to certain regions of the phase space may be large in comparison to the significance of these regions to the total energy. An example of such an operator is the Dirac delta function dependent on an interparticle distance. When Gaussian basis functions are used in the calculation, the expectation value of this operator is often significantly underestimated or overestimated. In such a situation the Dirac delta function may be determined several orders of magnitude less precisely than the total energy. However, the needed accuracy of the expectation values of the delta function may, in some cases, be quite high in order to yield adequately accurate values of such quantities as transition properties that depend on differences between expectation values of the delta function calculated for different states. Operators, whose expectation values converge slowly such as those of the delta function, also appear in the expressions for relativistic corrections and in $n$-photon decay rates. In this work we have attempted to perform calculations that yield not only very accurate total energies and wave functions but also can be used to evaluate expectation values of different operators with a very high precision.

The lowest variational energy upper bound to date for the
$\mathrm{Ps}_{2}$ ground state, according to our knowledge, remains that from the work of Usukura et al. [9], where the value of -0.516003789058 a.u. was reported. In that calculation the authors used 1600 explicitly correlated Gaussians (ECGs). For ${ }^{\infty}$ HPs (an infinitely heavy mass of the proton was assumed), the lowest energy of -0.789196740 a.u. was computed in Ref. [16] using 1800 ECG functions. In the case of the finite hydrogen mass ( $\left.{ }^{1} \mathrm{HPs}\right)$ the lowest energy value was obtained by the present authors [18] and it was -0.7888707066 a.u. In that work we used 3200 explicitly correlated Gaussian functions with premultipliers in the form of even powers of the distance between the proton and the positron.

In the calculations reported here we have used simple ECG functions (without premultipliers) in the following form:

$$
\phi_{k}=\exp \left[-\mathbf{r}^{\prime}\left(A_{k} \otimes I_{3}\right) \mathbf{r}\right]=\exp \left[-\mathbf{r}^{\prime}\left(L_{k} L_{k}^{\prime} \otimes I_{3}\right) \mathbf{r}\right] .
$$

In the above expression, $\mathbf{r}$ is a nine-component vector,

$$
\mathbf{r}=\left[\begin{array}{l}
\mathbf{r}_{1} \\
\mathbf{r}_{2} \\
\mathbf{r}_{3}
\end{array}\right]=\left[\begin{array}{l}
\mathbf{R}_{2}-\mathbf{R}_{1} \\
\mathbf{R}_{3}-\mathbf{R}_{1} \\
\mathbf{R}_{4}-\mathbf{R}_{1}
\end{array}\right],
$$

where the $\mathbf{R}_{i}$ 's are the original Cartesian coordinates of the particles in the laboratory coordinate system. $A_{k}$ is a symmetric, positive definite $3 \times 3$ matrix of exponential parameters that are unique for each basis function, $\otimes$ stands for the Kronecker product, and the prime is used to denote a matrix or a vector transposition. Explicitly correlated Gaussian functions (1) can also be written in a more conventional way;

$$
\begin{aligned}
\phi_{k}= & \exp \left[-\beta_{12, k} R_{12}^{2}-\beta_{13, k} R_{13}^{2}-\beta_{14, k} R_{14}^{2}-\beta_{23, k} R_{23}^{2}-\beta_{24, k} R_{24}^{2}\right. \\
& \left.-\beta_{34, k} R_{34}^{2}\right],
\end{aligned}
$$

where parameters $\beta_{i j, k}$ are related to the elements of matrices $A_{k}$ and $L_{k}$ in Eq. (1).

The choice of matrix $A_{k}$ in the Cholesky-factored form, $L_{k} L_{k}^{\prime}$, is convenient from a computational standpoint since this leads to no restrictions on the values of the elements of $L_{k}$. Such restrictions would have to be imposed if the elements of the $A_{k}$ matrices are used as the variational parameters since, as mentioned, $A_{k}$ 's have to be positive definite. More details describing the approach used here and those 
TABLE I. Energy convergence for $\mathrm{Ps}_{2},{ }^{1} \mathrm{HPs}$, and ${ }^{\infty} \mathrm{HPs}$. All values are in a.u.

\begin{tabular}{lccc}
\hline \hline Basis size & $\mathrm{Ps}_{2}$ & ${ }^{1} \mathrm{HPs}$ & ${ }^{\infty} \mathrm{HPs}$ \\
\hline 500 & -0.516003770835 & -0.788870158930 & -0.789196187887 \\
1000 & -0.516003787849 & -0.788870646568 & -0.789196705369 \\
2000 & -0.516003789829 & -0.788870702212 & -0.789196757758 \\
3000 & -0.516003790168 & -0.788870707806 & -0.789196762859 \\
4000 & -0.516003790332 & -0.788870709600 & -0.789196764467 \\
5000 & -0.516003790416 & -0.788870710444 & -0.789196765251 \\
\hline \hline
\end{tabular}

concerning the separation of the center-of-mass motion and evaluation of matrix elements with functions (1), etc., and those describing the computational procedures involved can be found in Refs. [19-21].

Before being used in the calculations, the basis functions (1) have to be transformed by symmetry operators to account for the permutational symmetry within each group of identical particles in the system. In the present calculations particles 3 and 4 were electrons, particle 2 was a positron, and particle 1 was either a proton (in HPs) or a positron (in $\mathrm{Ps}_{2}$ ). The proper symmetrization of the basis functions was achieved by applying to each basis function the $\left(1+\hat{P}_{34}\right)$ projection operator in the case of HPs and the $\left(1+\hat{P}_{13} \hat{P}_{24}\right)(1$ $\left.+\hat{P}_{12}\right)\left(1+\hat{P}_{34}\right)$ projection operator in the case of $\operatorname{Ps}_{2}$. Here $\hat{P}_{i j}$ stands for the permutation of particles $i$ and $j$. The result of applying the $\hat{P}_{i j}$ operators to basis functions (1) is equivalent to certain transformations of matrices $A_{k}$. There is an additional symmetry operator that needs to be applied to the basis functions for the $\mathrm{Ps}_{2}$ system that reflects the charge conjugation symmetry between the two electrons and the two positrons. This operator is $\left(1+\hat{P}_{13} \hat{P}_{24}\right)$ and it involves a permutation of the first electron with the first positron and a simultaneous permutation of the second electron with the second positron. A comprehensive analysis of the symmetry of the positronium molecule is given in Refs. [5,22].

In our calculations we used 5000 basis functions for each system. The basis set generation was carried out in two stages. In the first step we generated a small basis set of 100 functions and performed full optimization of all nonlinear parameters, i.e., all elements of the $L_{k}$ matrix, for each of the 100 basis functions. In this step we used the minimization procedure based on the analytical gradient of the energy calculated with respect to the $L_{k}$ parameters. Next the basis was enlarged to its final size of 5000 by incrementally adding 10-20 new functions to the basis set using a random selection of nonlinear parameters and optimizing one function at a time. After adding 10-20 functions the whole basis was reoptimized with the one-function-at-a-time approach. In this approach we also used the analytical gradient of the energy with respect to the exponential parameters. The formulas for the analytical gradient and remarks on its numerical implementation can be found in Refs. [19-21].

The convergence of the energies of the positronium molecule and the positronium hydride (for both finite and infinite proton masses) with respect to the number of the basis functions is shown in Table I. The proton-electron mass ratio used in the calculations of ${ }^{1} \mathrm{HPs}$ was 1836.15267261 . For all three systems we obtained the energy values that are the lowest energy upper bounds to date. Moreover, upon comparing the energies computed with different numbers of basis functions with the corresponding values taken from the works of Usukura et al. [9], Mitroy [16], and from our own work [18], one can notice that somewhat better energy convergence is achieved in the present calculations. This resulted from more thorough optimization of the exponential parameters performed in this work.

In order to determine the accuracy of the present calculations we examined the energy convergence patterns presented in Table I. Simple extrapolations gave us the values of $4 \times 10^{-10}$ and $2 \times 10^{-9}$ for the relative errors in the energies obtained with 5000 basis functions for $\mathrm{Ps}_{2}$ and HPs, respectively. The better convergence of the $\mathrm{Ps}_{2}$ energy results from the higher permutational symmetry of this system. This makes the number of the terms resulting from the symmetry

TABLE II. Expectation values for $\mathrm{Ps}_{2}$ obtained with different basis sets. All quantities are in a.u.

\begin{tabular}{|c|c|c|c|c|c|c|c|c|}
\hline $\begin{array}{l}\text { Basis } \\
\text { size }\end{array}$ & $\left\langle r_{e^{+} e^{+}}\right\rangle$ & $\left\langle r_{e^{+} e^{-}}\right\rangle$ & $\left\langle r_{e^{+} e^{+}}^{2}\right\rangle$ & $\left\langle r_{e^{+} e^{-}}^{2}\right\rangle$ & $\left\langle\delta_{e^{+} e^{+}}\right\rangle$ & $\left\langle\delta_{e^{+} e^{-}}\right\rangle$ & $\left\langle\delta_{e^{+} e^{+} e^{-}}\right\rangle$ & $\left\langle\delta_{e^{+} e^{+} e^{-} e^{-}}\right\rangle$ \\
\hline 500 & 6.033208400 & 4.487153726 & 46.37481041 & 29.11267056 & $6.267782 \times 10^{-4}$ & $2.210623 \times 10^{-2}$ & $9.16034 \times 10^{-5}$ & $4.5819 \times 10^{-6}$ \\
\hline 1000 & 6.033210193 & 4.487154574 & 46.37487478 & 29.11270241 & $6.260488 \times 10^{-4}$ & $2.211608 \times 10^{-2}$ & $9.13630 \times 10^{-5}$ & $4.5765 \times 10^{-6}$ \\
\hline 2000 & 6.033210239 & 4.487154592 & 46.37487781 & 29.11270393 & $6.259317 \times 10^{-4}$ & $2.211671 \times 10^{-2}$ & $9.11739 \times 10^{-5}$ & $4.5706 \times 10^{-6}$ \\
\hline 3000 & 6.033210196 & 4.487154570 & 46.37487660 & 29.11270337 & $6.257999 \times 10^{-4}$ & $2.211691 \times 10^{-2}$ & $9.10989 \times 10^{-5}$ & $4.5172 \times 10^{-6}$ \\
\hline 4000 & 6.033210225 & 4.487154583 & 46.37487782 & 29.11270397 & $6.257993 \times 10^{-4}$ & $2.211775 \times 10^{-2}$ & $9.11241 \times 10^{-5}$ & $4.5481 \times 10^{-6}$ \\
\hline 5000 & 6.033210257 & 4.487154599 & 46.37487910 & 29.11270461 & $6.257980 \times 10^{-4}$ & $2.211775 \times 10^{-2}$ & $9.10967 \times 10^{-5}$ & $4.5536 \times 10^{-6}$ \\
\hline
\end{tabular}




\begin{tabular}{|c|c|c|c|c|c|c|c|c|c|c|c|c|c|}
\hline $\begin{array}{l}\text { Basis } \\
\text { size }\end{array}$ & $\left\langle r_{p^{+} e^{+}}\right\rangle$ & $\left\langle r_{p^{+} e^{-}}\right\rangle$ & $\left\langle r_{e^{+} e^{-}}\right\rangle$ & $\left\langle r_{e^{-} e^{-}}\right\rangle$ & $\left\langle r_{p^{+} e^{+}}^{2}\right\rangle$ & $\left\langle r_{p^{+} e^{-}}^{2}\right\rangle$ & $\left\langle r_{e^{+} e^{-}}^{2}\right\rangle$ & $\left\langle r_{e^{-} e^{-}}^{2}\right\rangle$ & $\left\langle\delta_{p^{+} e^{+}}\right\rangle$ & $\left\langle\delta_{p^{+} e^{-}}\right\rangle$ & $\left\langle\delta_{e^{+} e^{-}}\right\rangle$ & $\left\langle\delta_{e^{-} e^{-}}\right\rangle$ & $\left\langle\delta_{e^{+} e^{-} e^{-}}\right\rangle$ \\
\hline 500 & 3.663475033 & 2.313145874 & 3.481162441 & 3.576992960 & 16.27171670 & 7.824557445 & 15.59328738 & 15.89547892 & $1.635665 \times 10^{-3}$ & 0.1767156 & $2.442215 \times 10^{-2}$ & $4.388081 \times 10^{-3}$ & $3.74685 \times 10^{-4}$ \\
\hline 1000 & 3.663501500 & 2.313160807 & 3.481175748 & 3.577021421 & 16.27214252 & 7.824786998 & 15.59353123 & 15.89592411 & $1.628128 \times 10^{-3}$ & 0.1769497 & $2.447620 \times 10^{-2}$ & $4.367616 \times 10^{-3}$ & $3.72553 \times 10^{-4}$ \\
\hline 2000 & 3.663502576 & 2.313161416 & 3.481176079 & 3.577022692 & 16.27217005 & 7.824801361 & 15.59354538 & 15.89595243 & $1.623934 \times 10^{-3}$ & 0.1770067 & $2.447765 \times 10^{-2}$ & $4.366199 \times 10^{-3}$ & $3.69374 \times 10^{-4}$ \\
\hline 3000 & 3.663502685 & 2.313161534 & 3.481176106 & 3.577022945 & 16.27217392 & 7.824804086 & 15.59354716 & 15.89595760 & $1.623818 \times 10^{-3}$ & 0.1770169 & $2.447774 \times 10^{-2}$ & $4.365969 \times 10^{-3}$ & $3.69200 \times 10^{-4}$ \\
\hline 4000 & 3.663502693 & 2.313161559 & 3.481176105 & 3.577023001 & 16.27217407 & 7.824804460 & 15.59354725 & 15.89595837 & $1.623825 \times 10^{-3}$ & 0.1770291 & $2.447780 \times 10^{-2}$ & $4.364226 \times 10^{-3}$ & $3.68791 \times 10^{-4}$ \\
\hline 5000 & 3.663502705 & 2.313161564 & 3.481176109 & 3.577023013 & 16.27217434 & 7.824804565 & 15.59354743 & 15.89595862 & $1.623808 \times 10^{-3}$ & 0.1770293 & $2.447844 \times 10^{-2}$ & $4.363357 \times 10^{-3}$ & $3.68779 \times 10^{-4}$ \\
\hline
\end{tabular}

TABLE IV. Expectation values for ${ }^{\infty}$ HPs obtained with different basis sets. All quantities are in a.u.

\begin{tabular}{|c|c|c|c|c|c|c|c|c|c|c|c|c|c|}
\hline Basis size & $\left\langle r_{p^{+} e^{+}}\right\rangle$ & $\left\langle r_{p^{+} e^{-}}\right\rangle$ & $\left\langle r_{e^{+} e^{-}}\right\rangle$ & $\left\langle r_{e^{-} e^{-}}\right\rangle$ & $\left\langle r_{p^{+} e^{+}}^{2}\right\rangle$ & $\left\langle r_{p^{+} e^{-}}^{2}\right\rangle$ & $\left\langle r_{e^{+} e^{-}}^{2}\right\rangle$ & $\left\langle r_{e^{-} e^{-}}^{2}\right\rangle$ & $\left\langle\delta_{p^{+} e^{+}}\right\rangle$ & $\left\langle\delta_{p^{+} e^{-}}\right\rangle$ & $\left\langle\delta_{e^{+} e^{-}}\right\rangle$ & $\left\langle\delta_{e^{-} e^{-}}\right\rangle$ & $\left\langle\delta_{e^{+} e^{-} e^{-}}\right\rangle$ \\
\hline 500 & 3.661598605 & 2.311511288 & 3.480259880 & 3.574759980 & 16.25402748 & 7.812796965 & 15.58399802 & 15.87498546 & $1.639838 \times 10^{-3}$ & 0.1770590 & $2.445015 \times 10^{-2}$ & $4.386228 \times 10^{-3}$ & $3.74881 \times 10^{-4}$ \\
\hline 1000 & 3.661623731 & 2.311525190 & 3.480272355 & 3.574786671 & 16.25451226 & 7.813037837 & 15.58426493 & 15.87545539 & $1.629110 \times 10^{-3}$ & 0.1772536 & $2.447607 \times 10^{-2}$ & $4.378337 \times 10^{-3}$ & $3.71128 \times 10^{-4}$ \\
\hline 2000 & 3.661624869 & 2.311525838 & 3.480272778 & 3.574787999 & 16.25453562 & 7.813049863 & 15.58427744 & 15.87547874 & $1.626244 \times 10^{-3}$ & 0.1773100 & $2.448248 \times 10^{-2}$ & $4.376174 \times 10^{-3}$ & $3.70262 \times 10^{-4}$ \\
\hline 3000 & 3.661624927 & 2.311525910 & 3.480272788 & 3.574788151 & 16.25453759 & 7.813051435 & 15.58427847 & 15.87548174 & $1.626068 \times 10^{-3}$ & 0.1773258 & $2.448364 \times 10^{-2}$ & $4.375841 \times 10^{-3}$ & $3.70351 \times 10^{-4}$ \\
\hline 4000 & 3.661624946 & 2.311525915 & 3.480272792 & 3.574788163 & 16.25453809 & 7.813051538 & 15.58427869 & 15.87548190 & $1.625320 \times 10^{-3}$ & 0.1773292 & $2.448374 \times 10^{-2}$ & $4.375389 \times 10^{-3}$ & $3.69835 \times 10^{-4}$ \\
\hline 5000 & 3.661624960 & 2.311525926 & 3.480272799 & 3.574788186 & 16.25453834 & 7.813051722 & 15.58427886 & 15.87548226 & $1.625230 \times 10^{-3}$ & 0.1773295 & $2.448406 \times 10^{-2}$ & $4.374170 \times 10^{-3}$ & $3.69456 \times 10^{-4}$ \\
\hline
\end{tabular}


projection for each basis function four times larger than in the case of HPs (eight vs two). It should be emphasized, however, that the above-mentioned relative energy errors are only rough estimates. For this reason we do not show any extrapolated energies in Table I.

The calculations of ${ }^{1}$ HPs and ${ }^{\infty}$ HPs have been performed independently from each other. Nonetheless, our tests showed that using the basis set generated for ${ }^{\infty} \mathrm{HPs}$ in the calculation for ${ }^{1} \mathrm{HPs}$, where only linear coefficients of the basis functions are optimized, works quite well. This is because the difference between the two systems, ${ }^{\infty} \mathrm{HPs}$ and ${ }^{1} \mathrm{HPs}$, is very small in terms of their structures and the optimization of only the linear coefficients suffices to get very good energy for ${ }^{1} \mathrm{HPs}$ with the ${ }^{\infty} \mathrm{HPs}$ basis set. In fact, the calculation of ${ }^{1} \mathrm{HPs}$ with the 5000 -term ${ }^{\infty} \mathrm{HPs}$ basis set gave slightly lower energy than that shown in Table I. The energy was -0.788870710567 a.u. vs -0.788870710444 a.u. given in Table I. The likely reason for this is the fact that the calculations for ${ }^{\infty} \mathrm{HPs}$ were slightly better converged than those for ${ }^{1} \mathrm{HPs}$ and produced a better basis set even for ${ }^{1} \mathrm{HPs}$ (growing the basis up to 5000 and optimizing the nonlinear parameters is, in some way, a trial and error process).

In Tables II-IV we present some expectation values computed with progressively larger basis sets. The expectation values of the interparticle distances and their squares for all systems show convergence that is comparable with the convergence of the total energy (they are about one order of magnitude less accurate). These values are in very good agreement with the corresponding data from works $[9,16]$. We also computed the expectation values of the two-particle contact densities, as well as three- and four-particle electronpositron contact densities. They can be used to make estimates of various positron-electron annihilation rates. The notation used in Tables II-IV is the following: $\left\langle\delta_{e^{+} e^{-}}\right\rangle \equiv\left\langle\delta\left(\mathbf{r}_{e^{+}}-\mathbf{r}_{e^{-}}\right)\right\rangle, \quad\left\langle\delta_{e^{+} e^{+} e^{-}}\right\rangle \equiv\left\langle\delta\left(\mathbf{r}_{e^{+}}-\mathbf{r}_{e^{+}}\right) \delta\left(\mathbf{r}_{e^{+}}-\mathbf{r}_{e^{-}}\right)\right\rangle$, $\left\langle\delta_{e^{+} e^{+} e^{-} e^{-}}\right\rangle \equiv\left\langle\delta\left(\mathbf{r}_{e^{+}}-\mathbf{r}_{e^{+}}\right) \delta\left(\mathbf{r}_{e^{+}}-\mathbf{r}_{e^{-}}\right) \delta\left(\mathbf{r}_{e^{-}}-\mathbf{r}_{e^{-}}\right)\right\rangle$. As expected, the relative accuracy of the contact densities is significantly lower than that of the total energy. The two-particle contact densities for particles with opposite charges tend to be slightly underestimated, while the contact densities for particles with the same charge are usually overestimated. This results from the well-known deficiency of the Gaussian functions and their inability to describe the cusp behavior of the wave function. The relative accuracy drops even further for the three- and four-particle contact densities.

Using the contact-density data obtained in the calculations with the largest basis sets, we computed the zero-, one-, two-, and three-photon annihilation rates. The two-photon annihilation rate for a system containing both electrons and positrons, which is the dominant rate for this type of system, can be computed using the following expression that involves the expectation value of the electron-positron contact density:

$$
\Gamma_{2 \gamma}=N_{e^{+}} N_{e^{-}} \frac{\pi \alpha^{4} c}{a_{0}}\left\langle\delta_{e^{+} e^{-}}\right\rangle .
$$

Here $\alpha$ is the fine structure constant, $a_{0}$ is the Bohr radius, and $c$ is the velocity of light. $N_{e^{+}}$and $N_{e^{-}}$denote the number of positrons and electrons in the system, respectively. Taking the expectation values of $\delta_{e^{+} e^{-}}$calculated with 5000 basis functions, one obtains the two-photon annihilation rates of $4.465106 \times 10^{9} \mathrm{~s}^{-1}, \quad 2.470839 \times 10^{9} \mathrm{~s}^{-1}$, and 2.471406 $\times 10^{9} \mathrm{~s}^{-1}$ for $\mathrm{Ps}_{2}{ }^{1} \mathrm{HPs}$, and ${ }^{\infty} \mathrm{HPs}$, respectively. It is possible to correct $\Gamma_{2 \gamma}$ for the Coulomb and the radiative effects [23], as it was done in Ref. [24]. In order to do this, one needs to multiply the expression (4) by the factor $1-\alpha\left(5-\pi^{2} / 4\right) / \pi$. The annihilation rates corrected in this way are 4.438838 $\times 10^{9} \mathrm{~s}^{-1}, \quad 2.456304 \times 10^{9} \mathrm{~s}^{-1}, \quad$ and $2.456867 \times 10^{9} \mathrm{~s}^{-1}$ (again, for $\mathrm{Ps}_{2},{ }^{1} \mathrm{HPs}$, and ${ }^{\infty} \mathrm{HPs}$, respectively). The above $\mathrm{Ps}_{2}$ two-photon annihilation rate value differs from the one given in Ref. [24] because the authors of that work mistakenly used $\pi^{4}$ instead of $\pi^{2}$ in the expression for the correction factor.

Following the works $[8,24,25]$ we can also evaluate the one- and three-photon annihilation rates. The corresponding expressions are

$$
\begin{gathered}
\Gamma_{1 \gamma}=k N_{e^{+}} N_{e^{-}} \frac{32 \pi^{2} \alpha^{8} c}{27 a_{0}}\left\langle\delta_{e^{+} e^{+} e^{-}}\right\rangle, \\
\Gamma_{3 \gamma}=N_{e^{+}} N_{e^{-}} \frac{4\left(\pi^{2}-9\right) \alpha^{5} c}{3 a_{0}}\left\langle\delta_{e^{+} e^{-}}\right\rangle .
\end{gathered}
$$

The coefficient $k$ in Eq. (5) should be set to 1 for $\mathrm{Ps}_{2}$ and to 2 for HPs because in the latter system there are two ways the electron-positron annihilation can occur and both ways have approximately equal annihilation rates [8].

The numerical values for the one- and three-photon annihilation rates obtained using the expectation values from Tables II-IV are $\Gamma_{1 \gamma}\left(\mathrm{Ps}_{2}\right)=0.194174 \mathrm{~s}^{-1}, \Gamma_{1 \gamma}\left({ }^{1} \mathrm{HPs}\right)$ $=0.786058 \mathrm{~s}^{-1}, \quad \Gamma_{1 \gamma}{ }^{\left({ }^{\infty} \mathrm{HPs}\right)}=0.787501 \mathrm{~s}^{-1}, \quad \Gamma_{3 \gamma}\left(\mathrm{Ps}_{2}\right)$ $=1.202563 \times 10^{-7} \mathrm{~s}^{-1}, \quad \Gamma_{3 \gamma}\left({ }^{1} \mathrm{HPs}\right)=6.654577 \times 10^{-6} \mathrm{~s}^{-1}$, $\Gamma_{3 \gamma}\left({ }^{\infty} \mathrm{HPs}\right)=6.656103 \times 10^{-6} \mathrm{~s}^{-1}$.

For the positronium molecule, a zero-photon annihilation process (two-photon annihilation of a $e^{+} e^{-}$pair followed by the internal conversion of the emitted photons) can also occur. The expression for the annihilation rate for such a process as given in Ref. [24] is

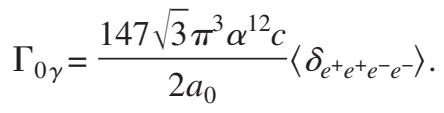

Using the $\left\langle\delta_{e^{+} e^{+} e^{-} e^{-}}\right\rangle$expectation value taken from Table II we get $\Gamma_{0 \gamma}=2.3220 \times 10^{-9} \mathrm{~s}^{-1}$.

In summary, we have performed very accurate groundstate nonrelativistic calculations of the positronium molecule and the positronium hydride using explicitly correlated Gaussian functions. The computed energies set new improved variational energy upper bounds for these systems. The obtained wave functions can be used in accurate calculations of expectation values of various operators, in particular, of operators that appear in the calculations of relativistic corrections.

This work has been supported in part by the National Science Foundation. We would also like to thank University of Arizona Center of Computing and Information Technology for the use of their supercomputer resources. 
[1] E. A. Hylleraas and A. Ore, Phys. Rev. 71, 493 (1947).

[2] A. Ore, Phys. Rev. 83, 665 (1951).

[3] S. M. Neamtan, G. Darewych, and G. Oczkowski, Phys. Rev. 126, 193 (1962).

[4] Y. K. Ho, Phys. Rev. A 33, 3584 (1986).

[5] D. B. Kinghorn and R. D. Poshusta, Phys. Rev. A 47, 3671 (1993).

[6] P. M. Kozlowski and L. Adamowicz, Phys. Rev. A 48, 1903 (1993).

[7] T. K. Rebane, V. S. Zotev, and O. N. Yusupov, Zh. Eksp. Teor. Fiz. 110, 55 (1996).

[8] A. M. Frolov and V. H. Smith, Phys. Rev. A 55, 2662 (1997).

[9] J. Usukura, K. Varga, and Y. Suzuki, Phys. Rev. A 58, 1918 (1998).

[10] K. Varga, J. Usukura, and Y. Suzuki, Phys. Rev. Lett. 80, 1876 (1998).

[11] T. K. Rebane and N. D. Markovskii, Opt. Spectrosc. 89, 667 (2000).

[12] Y. K. Ho, Phys. Rev. A 34, 609 (1986).
[13] D. Bressanini, M. Mella, and G. Morosi, Phys. Rev. A 57, 1678 (1998).

[14] Z.-C. Yan and Y. K. Ho, Phys. Rev. A 60, 5098 (1999).

[15] J. Usukura and Y. Suzuki, Phys. Rev. A 66, 010502(R) (2002).

[16] J. Mitroy, Phys. Rev. A 73, 054502 (2006).

[17] D. M. Schrader, F. M. Jacobsen, N. P. Frandsen, and U. Mikkelsen, Phys. Rev. Lett. 69, 57 (1990).

[18] S. Bubin and L. Adamowicz, J. Chem. Phys. 120, 6051 (2004).

[19] S. Bubin, M. Cafiero, and L. Adamowicz, Adv. Chem. Phys. 131, 377 (2005).

[20] D. B. Kinghorn, Int. J. Quantum Chem. 57, 141 (1996).

[21] S. Bubin and L. Adamowicz, J. Chem. Phys. 124, 224317 (2006).

[22] D. M. Schrader, Phys. Rev. Lett. 92, 043401 (2004).

[23] I. Harris and L. M. Brown, Phys. Rev. 105, 1656 (1957).

[24] D. H. Bailey, A. M. Frolov, Phys. Rev. A 72, 014501 (2005).

[25] A. M. Frolov, S. I. Kryuchkov, and V. H. Smith, Phys. Rev. A 51, 4514 (1995). 